\title{
Forbidden reflections from the aramid PPTA-A novel correlation with stacking faults
}

\author{
ANJANA JAIN and KALYANI VIJAYAN* \\ Materials Science Division, National Aerospace Laboratories, Bangalore 560 017, India
}

MS received 23 August 2003

\begin{abstract}
The occurrence of space group forbidden reflections in the X-ray diffraction patterns from the aramid PPTA has been correlated with the presence of stacking faults. The fraction of sample affected by the presence of such faults has also been estimated.
\end{abstract}

Keywords. Stacking fault; X-ray diffraction; Kevlar; Twaron.

\section{Introduction}

The high strength, high modulus, low-density fibres, Kevlar and Twaron, are both made of poly ( $p$-phenylene terephthalamide) or (PPTA) (Fitzer et al 1986), the structural formula of which has been presented below.

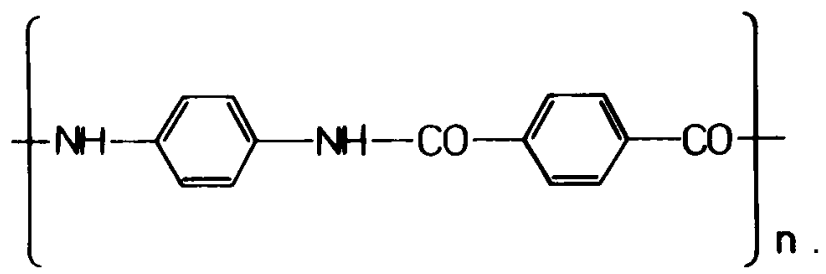

Structural studies have shown that PPTA crystallizes in the monoclinic crystal system (Northolt 1974; Tashiro et al 1977). With a monoclinic angle of $\approx 90^{\circ}$, the crystal system may also be described as pseudo orthorhombic. Although the crystal system to which PPTA belongs is known unambiguously, there has been some controversy about the space group. The controversy centres around the presence or absence of an $n$-glide. Table 1 lists information concerning two sets of space groups viz. I and II, reported for PPTA. Basically the two sets refer to space groups with and without an $n$-glide respectively, irrespective of the crystal system.

The stable form of PPTA, henceforth referred to as modification I, crystallizes in the monoclinic space group, $P n$ and $P 2_{1} / n$ i.e. set I. It must be mentioned that to carry out ab initio simulations, Rutledge and Suter (1991) started with triclinic unit cell, which belong to set II (table 1). However, their calculations eventually converged on the monoclinic modification I with an $n$-glide thus suggesting the enhanced stability associated with a space group with an $n$-glide.

\footnotetext{
*Author for correspondence
}

An unstable modification (II) of PPTA crystallizing in the monoclinic crystal system has also been reported by Haraguchi et al (1979). The space group, $\mathrm{Pa}$, reported for this modification lacks an $n$-glide and therefore belongs to set II. They however, mention that on annealing, modification II transforms to the stable form characterized by the presence of an $n$-glide. This observation also suggests that for PPTA, space group with $n$-glide, is perhaps the more natural choice. The more recent work of Liu et al (1996), however, differs from the earlier observations and it suggests the space group to be $\mathrm{Pa}$. It must be pointed out that irrespective of the presence or absence of an $n$-glide, the unit cell dimensions and the fully extended all trans molecular conformation reported by various workers, for various forms of PPTA, viz. fibres, films etc are nearly the same.

In set I, presence of an $n$-glide perpendicular to the crystallographic $c$-axis (unique axis of the monoclinic unit cell), necessitates systematic absence or forbidden reflections of the type $(h k 0), h+k$ odd in the diffraction pattern. Although the crystal structure determination of PPTA reported by Northolt is based on the space group $P n$ or $P 2{ }_{1} / n$, he mentions about the occurrence of few of the forbidden $(h k 0)$ reflections with $(h+k)$ odd, in the $\mathrm{X}$ ray diffraction patterns from fibres heat treated at $400^{\circ} \mathrm{C}$ (Northolt 1974). Liu et al (1996) also report the occurrence of similar forbidden reflections in the electron diffraction patterns from heat-treated crystals of PPTA. Conspicuously, the forbidden reflections mentioned by both Northolt and Liu et al are from heat-treated samples. The present study, in contrast, concerns the observation of forbidden reflections in the X-ray diffraction patterns from commercially available Kevlar 49 and Twaron fibres, both prior to and after heat treatment. This paper proposes a novel method of explaining the anomalous occurrence of the $(h k 0), h+k$ odd reflections in the diffraction patterns from PPTA, on the basis of a single phase defect model, without recourse to a space group in set II. The 
model is based primarily on the crystal structural characteristics of the stable form of PPTA.

\section{Experimental}

Equatorial X-ray diffraction patterns were recorded from Kevlar 49 and Twaron fibres, commercialized by DuPont Inc., USA and Akzo Nobel, The Netherlands, respectively using the fibre attachment on a D/MAX 2200 Ultima $\mathrm{X}$-ray diffractometer. $\mathrm{CuK} \alpha$ radiation, scintillation counter and graphite monochromator in the diffracted beam were employed. Integrated intensities were estimated from the area under the diffraction profiles using a digitizer and an Autocad system.

\section{Results and discussion}

Figures 1(a) and (b) present the equatorial patterns from Kevlar 49 and Twaron fibres, respectively. The anomalous reflections with $h+k$ odd values have been identified by an asterisk mark ' $*$ '. Interestingly, the $(h+k)$ odd reflections are all uniformly weak in intensity and are also not sharp. The reliability of occurrence of these weak reflections has been confirmed by recording the X-ray patterns from different samples. Although low in intensity and seen only as a small hump above the background, these reflections were found to recur in the patterns from different samples. Of the four $(h+k)$ odd reflections which are observed (figure 1), (210) is the most intense. As the intensity distribution in the rest of the pattern conformed well with what was expected from PPTA (Northolt 1974), it appeared appropriate to assume that, basically, the diffracting materials had the crystal structure proposed by Northolt viz. corresponding to a space group with an $n$ glide. If so, how could the forbidden reflections with $(h+k)$ odd indices be observed? The simplest explanation is to consider the diffracting material as made up of two phases, the major one belonging to set I and a minor fraction from set II. This paper presents a different approach in which a single phase defect model for PPTA has been proposed. Admittedly, at this stage, the model is only qualitative in nature.

PPTA is characterized by a layer structure (Northolt 1974; Tashiro et al 1977). Figures 2(a) and (b) depict the molecular arrangements in the crystallographic $b c$ - and $a b$-planes, respectively. The former depicts the layer structure which is stabilized primarily by a network of inter chain NH...O hydrogen bonds. Such hydrogen bonded layers are stacked regularly along the crystallographic $a$-direction. By virtue of the $n$-glide in the space group $P n$ or $P 2_{1} / n$, adjacent layers along the $a$-direction are staggered with respect to each other by the $n$-glide translation of $a / 2+b / 2$. Figure 2(b) illustrates this feature. Here, the polymer chain is oriented in the direction perpendicular to the plane of the paper and is contained in the crystallographic $b c$-plane.

The arrangement shown in figure 2(b) can be described as a periodic, infinite stacking of two sets of parallel layers marked as $A$ and $B$, respectively. The molecular arrangements in both $A$ and $B$ layers are as in figure 2(a). Structurally, adjacent layers $A$ and $B$ are related by the $n$-glide. Based on this notation, the crystal structure may be described as a regular stacking of two types of layers viz. $A B A B A B$. ., along the crystallographic $a$-direction. In this sequence, the identities of the two sets of layers, $A$ and $B$, are interchangeable and each layer is same as the second one on either side. The $A B A B A B \ldots$ stacking arrangement is stabilized, primarily, by van der Waal's and $\pi$-electron interactions between adjacent layers. It must be emphasized that the presence of an $n$-glide dictates the staggered positions of adjacent layers $A$ and $B$. If the space group did not include an $n$-glide, the staggered molecular arrangement in layers $A$ and $B$ shown in figure 2(b) will not exist.

Table 1. Space group and relevant crystal data on PPTA.

\begin{tabular}{|c|c|c|c|}
\hline Sample & $\begin{array}{l}\text { Space } \\
\text { group sets }\end{array}$ & Molecular orientation & References \\
\hline Fibre & I & $\begin{array}{l}\text { Chains oriented along the } \\
\text { unique } \operatorname{axis}(c)\end{array}$ & Northolt (1974) \\
\hline Fibre & I & "I & Tashiro et al (1977) \\
\hline Film & II & $"$ & Haraguchi et al (1979) \\
\hline $\begin{array}{l}\text { Single crystal and } \\
\text { negative spherulites }\end{array}$ & Not mentioned & $"$ & Li (1986) \\
\hline $\begin{array}{l}\text { Fibre - As spun } \\
\text { - Annealed } \\
- \text { Simulation }\end{array}$ & $\begin{array}{c}\text { II } \\
\text { I }\end{array}$ & " & Rutledge et al (1991) \\
\hline $\begin{array}{l}\text { - Simulation } \\
\text { Crystal }\end{array}$ & $\begin{array}{l}\text { I and II } \\
\text { I }\end{array}$ & " & Jackson and Chanzy (1993) \\
\hline Single crystal & II & $\begin{array}{l}\text { Chains not oriented along } \\
\text { unique axis }(b)\end{array}$ & Liu et al (1996) \\
\hline
\end{tabular}

${ }^{\$}$ Sets I and II refer to space groups with and without $n$-glide, respectively. 
Stacking fault is a well known defect in metallic materials. For example, in the case of $f c c$ structures, along the $<111>$ direction, the stacking sequence is of the type

$$
A B C A B C A B C \text {. . }
$$

A break in the sequence of the type indicated below

$$
A B C \downarrow B C A B C . .
$$

is a deformation fault (Barrett 1952; Warren 1968; Frank and Steeds 1975). Here, the symbol $\downarrow$ refers to a fault plane. Indeed the ease of occurrence of a fault depends on the associated energy. If the stacking fault energy is low, the fault occurs readily. Extending the above mentioned concept of faulting to the layer sequence described for PPTA, it appears that a faulting of the type indicated below can be expected.

$$
A B A \downarrow A B \ldots
$$

As the interaction between adjacent layers in the crystal structure of PPTA is primarily of the van der Waal's type, the probability of occurrence of such faultings may also be expected to be not too low. As mentioned earlier, the staggered disposition of adjacent layers $A$ and $B$ is dictated by the presence of $n$-glide. Therefore, a faulting
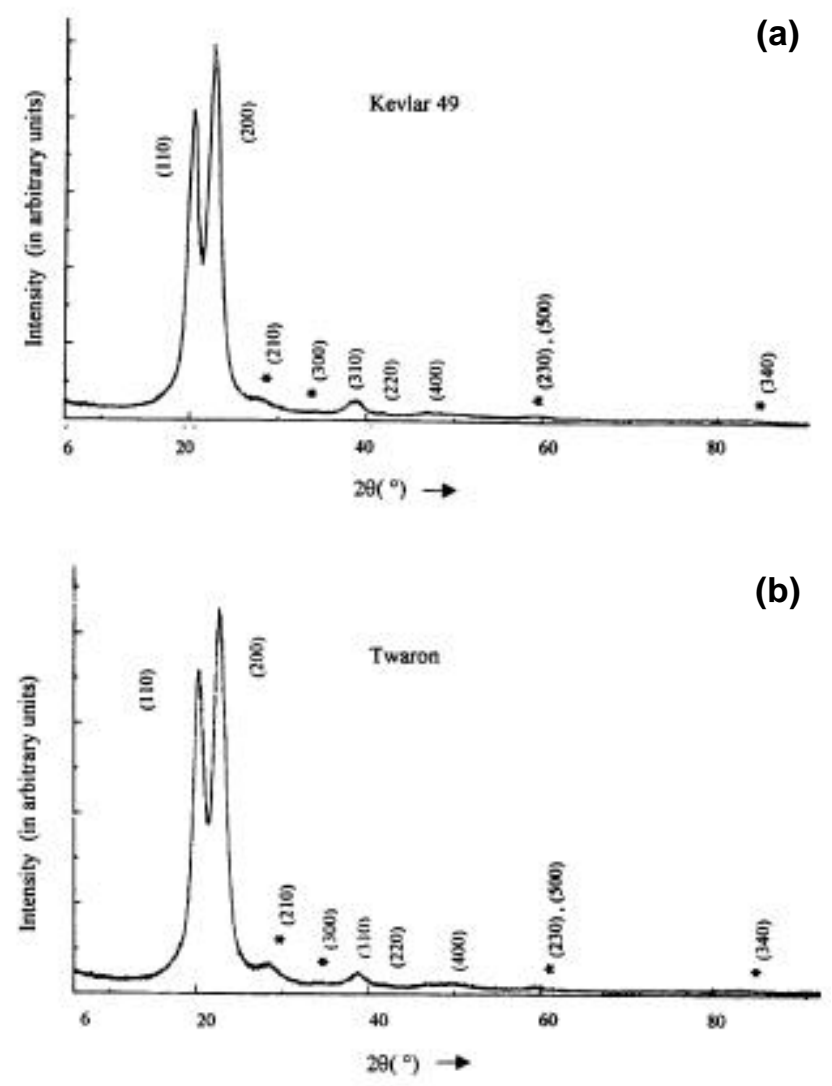

Figure 1. Equatorial X-ray diffraction patterns from (a) Kevlar 49 and (b) Twaron fibres. The forbidden reflections have been identified by the $*$ mark. of the type shown above tantamounts to a deviation from the $n$-glide imposed arrangement of layers. The crystallographic implication of a fault, therefore, is the absence of $n$-glide in that part of the crystal structure. It may be expected that when faults are sufficiently large in number, reflections, which are normally forbidden by the $n$-glide, may be expected to appear in the diffraction pattern. The $n$-glide forbidden reflections denoted by the symbol $*$ in the diffraction patterns in figures $1(a)$ and (b) may thus be considered as manifestation of the presence of faultings in the $A B A B \ldots$. type of sequence.

The effect of growth faults on X-ray diffraction patterns from metallic materials has been a topic extensively discussed in text books (Barrett 1952; Warren 1968;

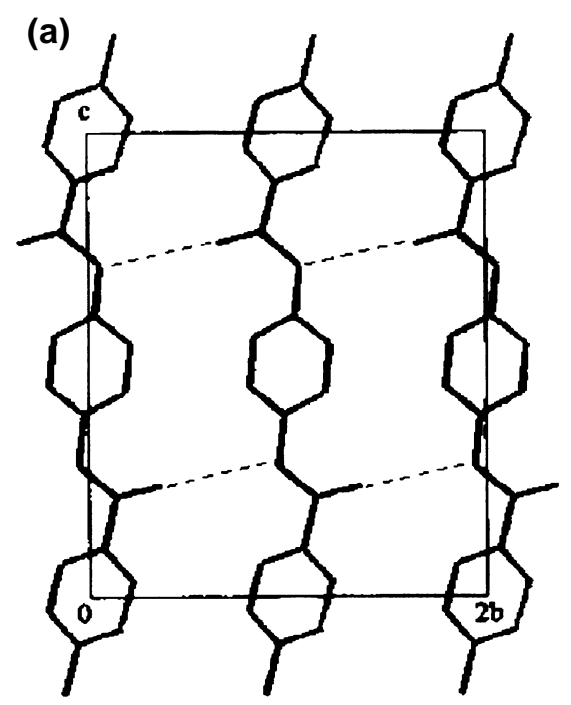

(b)

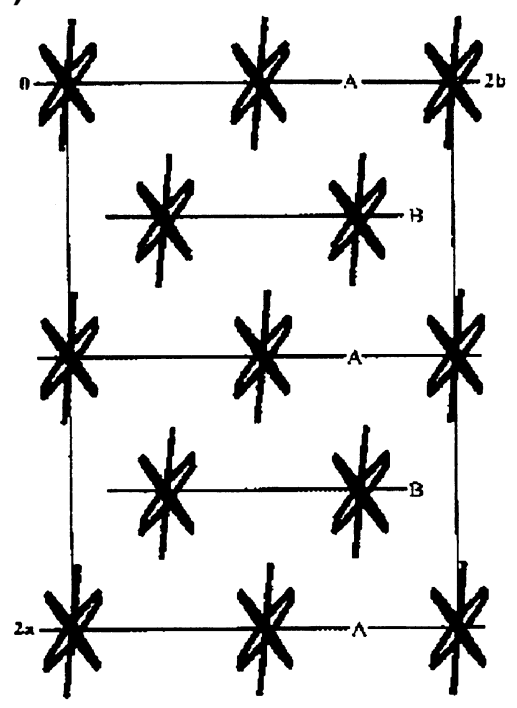

Figure 2. (a) Molecular arrangement in the crystallographic $b c$-plane. The dashed lines denote the interchain NH...O hydrogen bonds and (b) molecular arrangement in the crystallographic $a b$-plane. $A$ and $B$ denote the layers. 
Frank and Steeds 1975). The available information for estimation of stacking fault probability, stacking fault energy etc from fault induced shifts and asymmetry of diffraction profiles are, however, concerned with high symmetry crystal systems such as cubic or hexagonal in which most metallic materials crystallize. For lower symmetry systems such as monoclinic to which PPTA belongs, the mathematical procedures for deducing quantitative information on stacking fault probability or energy are not equally well known. Conclusive evidence for the proposed defect model can also be obtained from simulations in which the diffraction intensities are calculated on the basis of the defect structure. However, at this stage of the study, due to the non availability of appropriate software, estimates of neither the stacking fault energy, probability nor the diffraction intensities could be made. Nevertheless, a very rough estimate of the fraction of the sample affected by the presence of faults has been obtained from the intensity ratio

\section{$\Sigma I$ (forbidden reflections)/I (entire pattern).}

The values of $2.8 \%$ and $3.1 \%$ deduced for Kevlar and Twaron, respectively from the above mentioned ratio suggest that along the $A B A B \ldots$ layer stacking direction, $\sim 30$ in every 1000 layers are likely to be faulted. This value is indeed sufficient to manifest as forbidden reflections in the diffraction pattern. Observation of the forbidden reflections in the patterns from Kevlar and Twaron both of which are made of PPTA, suggests that presence of faults is perhaps an inherent structural characteristic of the PPTA fibre, facilitated by the crystal structural characteristics and perhaps also by the processing conditions and not dependent on the source.

When synthetic polymers with high molecular weight are crystallized, it is common that defects of various types get included. In the case of Kevlar fibres, inclusion of defects in the form of voids (Dobb et al 1979; Lee et al 1985), salts (Penn and Larsen 1979; Morgan and Pruneda 1987; Vijayan 1987) etc are well established. Panar et al (1983) treat the bends in the pleated structure which characterizes Kevlar fibres (Dobb et al 1979; Simmens and Hearle 1980; Allen and Roche 1989) also as a form of defect. The present study suggests that in addition to these defects, faulting in the stacking sequence of layers in the crystal structure may also be included in the category of defects.

The concept of faulting is commonly associated with metallic materials and is comparatively less frequently used in the case of organic materials like Kevlar/Twaron. It must be emphasized that faulting leading to the occurrence of space group forbidden reflections, as observed in the case of PPTA, is case-specific and cannot indeed be generalized. In the case of metallic materials, the common manifestations of faults are shifts in $2 \theta$ values and introduction of asymmetry of profiles. Forbidden reflections can occur only when the sequence of stacking is related to the space group symmetry, as in the case of PPTA and cannot be generalized to all materials which can have stacking faults.

\section{Conclusions}

The anomalous occurrence of space group forbidden reflections in the X-ray diffraction patterns from the aramid PPTA has been explained qualitatively on the basis of a single phase defect model, without recourse to a space group other than $P n$ or $P 2_{1} / n$. It is proposed that faulting in the stacking of layers in the crystal structure is responsible for the occurrence of space group forbidden reflections.

\section{Acknowledgement}

The authors thank Dr R V Krishnan, Materials Science Division, NAL, Bangalore, for the support.

\section{References}

Allen S R and Roche E J 1989 Polymer 30996

Barrett C S 1952 Structure of metals (NewYork: McGraw-Hill) Ch. 11

Dobb M G, Johnson D J, Majeed A and Saville B P 1979 Polymer 201284

Fitzer E, Kompalik D and Kunz M 1986 Deutsche Keramischen Gesellschaft E.V. 847

Frank F C and Steeds J W 1975 The physics of metals 2. Defects (ed.) P B Hirsch (Cambridge: Cambridge University) Ch. 2

Haraguchi K, Kajiyama T and Takayanagi M 1979 J. Appl. Polym. Sci. 23 903, 915

Jackson C L and Chanzy H D 1993 Polymer 345011

Lee J S, Fellers J S, Tang M Y and Lin J S 1985 J. Comp. Mater. 19114

Li L S 1986 J. Macromol. Sci., Phys. B25 215

Liu J, Cheng S Z D and Geil P H 1996 Polymer 371413

Morgan R J and Pruneda C O 1987 Polymer 28340

Northolt M G 1974 Eur. Polym. J. 10799

Panar M, Avakian A, Blume R C, Gardner K H, Gierke T D and Yang H H 1983 J. Polym. Sci., Polym. Phys. Ed. 211955

Penn L and Larsen F 1979 J. Appl. Polym. Sci. 2359

Rutledge G C and Suter U W 1991 Macromolecules 241921

Rutledge G C, Suter U W and Papaspyrides C D 1991 Macromolecules 241934

Simmens S C and Hearle J W S 1980 J. Polym. Sci., Polym. Phys. Ed. 18871

Tashiro K, Kobayashi M and Tadokoro H 1977 Macromolecules 10413

Vijayan K 1987 Curr. Sci. 561055

Warren B E 1968 X-ray diffraction (London: Addison-Wesley) p. 277 